\title{
Calculation of kinematic parameters of a flat mechanism
}

\author{
Smirnova,ZhannaV. ${ }^{1}$ VaganovaO.I. ${ }^{2}$ GruzdevaM.L. ${ }^{3}$ Smirnov A. B. ${ }^{4}$ \\ ${ }^{1}$ Minin Nizhny Novgorod State Pedagogical University (Minin University), Nizhny Novgorod, Russian Federation, e-mail: \\ z.v.smirnova@mininuniver.ru \\ ${ }^{2}$ Minin Nizhny Novgorod State Pedagogical University (Minin University), Nizhny Novgorod, Russian Federation, e-mail: \\ vaganova_o@mail.ru \\ ${ }^{3}$ Minin Nizhny Novgorod State Pedagogical University (Minin University), Nizhny Novgorod, Russian Federation, e-mail: \\ gru1234@yandex.ru \\ ${ }^{4}$ Minin Nizhny Novgorod State Pedagogical University (Minin University), Nizhny Novgorod, Russian Federation, e-mail: \\ smirnov_ab@mininuniver.ru
}

\begin{abstract}
Innovative technologies in the construction industry today have widely taken a confident position in the construction industry. The article discusses new technologies used in the work to strengthen the foundations of various kinds. A study was conducted on the application of the traditional way of strengthening foundations and indicators of the use of new technologies. Traditional options for strengthening the foundations associated with increasing the area of the sole are considered from the standpoint of geotechnics and manufacturability in relation to weak waters with saturated soils. The technological characteristics of the results of new technologies during the work on strengthening the foundations are given.
\end{abstract}

Keywords:building maintenance, foundation, foundation strengthening

\section{INTRODUCTION}

The theory of mechanisms and machines (TMM) is based on fundamental knowledge in the study of mathematics, physics, theoretical mechanics and is the basis for the study of subsequent practical (special) disciplines. The main issues that TMM is studying include:

- study of the structure (structure) of mechanisms;

- determination of the positions of mechanisms and trajectories described by individual points;

- determination of speeds and accelerations of individual points;

- analysis and design of various mechanisms (gear, cam);

- determination of different forces (external, reactions, friction, inertia) acting on the mechanism;

- the study of the energy balance of machines (efficiency);

- the study of the actual law of motion of machines under the action of given forces;

- the study of ways to control the speed of the machine;

- the study of methods of balancing the forces of inertia in machines, etc [2].

Thus, we can say that the theory of mechanisms and machines is a science that studies the structure, kinematics and dynamics of mechanisms and machines.
The purpose of this study is to conduct a kinematic analysis of the planar mechanism and consolidate the theoretical knowledge obtained in the study of TMM.

Getting to the analysis of the existing or the synthesis of a new mechanism, it is necessary, first of all, to determine its structure, that is, to draw up a kinematic diagram, count the number of moving links, determine the nature of their connection with each other, understand the method of formation of the mechanism and clearly imagine the scheme of transmission of motion from leading links to followers.

\section{RESEARCH}

Kinematic study of the mechanism, i.e. the study of the movement of the links of the mechanism without taking into account the forces that determine this movement consists mainly in solving three problems:

1. The determination of the movements of links and trajectories described by points of links.

2. The determination of the speeds of the individual points of the links and the angular velocities of the links.

H. Determination of accelerations of individual points of links and angular accelerations of links [1].

The determination of the movements of links and trajectories described by the points of the links makes it possible to analyze the correct action of the mechanism, the correspondence of the trajectories of the working bodies of the machine to the technological processes for which they are intended, and also to determine the space required to accommodate the mechanism. Knowledge of the values of the speed of movement of the links and their points is necessary to determine the kinetic energy of individual links and the mechanism as a whole when solving problems of machine dynamics. From the motion acceleration vectors, the magnitudes and directions of the inertia forces are determined, and therefore, the loads applied to the details of the mechanisms.

To perform a kinematic analysis of the movement of the links of the mechanism, the following must be specified: the kinematic diagram of the mechanism, the dimensions of its links, as well as the functional dependence of the movements of the leading links on the time parameter or on other parameters of their movement [3]. 
There are three main methods of kinematic study of mechanisms:

1. The method of graphs (least accurate and least laborintensive).

2. The method of plans (more accurate and more timeconsuming).

3. The analytical method (the most accurate and the most time-consuming).

4. The graphical method, based on the construction of graphs of the laws of motion with the use of graphical differentiation, has simplicity and clarity, but has insufficient accuracy, therefore, the graphic-analytical method is used in engineering calculations.

It gives satisfactory accuracy, but requires accurate execution of graphic work and compliance with scale [8].

By scale is meant the ratio of the actual value, expressed in appropriate units, to the length of the segment depicting this value, expressed in millimeters. When constructing kinematic schemes and plans of the positions of mechanisms, a length scale is determined that shows the number of meters of a natural size corresponding to one millimeter of the drawing $(\mathrm{m} / \mathrm{mm})$ :

$$
\mu_{l}=\frac{l_{O A}}{O A},
$$

where $\quad l_{O A}-$ actual crank length $(\mathrm{m})$;

$\mathrm{OA}$ - the length of the segment depicting the crank in the drawing (mm).

When building speed and acceleration plans in the drawing, the values of speed and acceleration are postponed to a certain scale.

Point calculated speed vector $\overline{\mathrm{V}}_{\mathrm{A}}(\mathrm{m} / \mathrm{s})$ on the speed plan is depicted as a segment $\overline{p a}$ arbitrary length $(\mathrm{mm})$. To find the scale of the speed plan $\left(\mathrm{m} / \mathrm{s}-\mathrm{mm}^{-1}\right)$, a speed value is necessary $\mathrm{V}$ A divided by the length of this segment:

$$
\mu_{v}=\frac{v_{A}}{\overline{p a}}(2)
$$

Similarly, the scale of the acceleration plan $\left(\mathrm{m} / \mathrm{s}-\mathrm{mm}^{-1}\right)$

$$
\text { is found: } \mu_{a}=\frac{a_{A}}{\overline{p a}}(3)
$$

where $\alpha \mathrm{A}$ - the calculated value of the acceleration of point $\mathrm{A}\left(\mathrm{m} / \mathrm{s}^{2}\right)$;

- the scale value of the acceleration of point A (mm).

The true values of the speed and acceleration of any point on the mechanism are obtained from their scale values by multiplying the latter by an appropriate scale [5].

An important kinematic characteristic in the synthesis of a mechanism is the condition for the rotation of its links (the presence of one or two cranks in it), which depends on the ratio of the lengths of the links [6].

This condition means that there must be one or two cranks in the linkage, that is, a link capable of making a complete revolution around a fixed point.

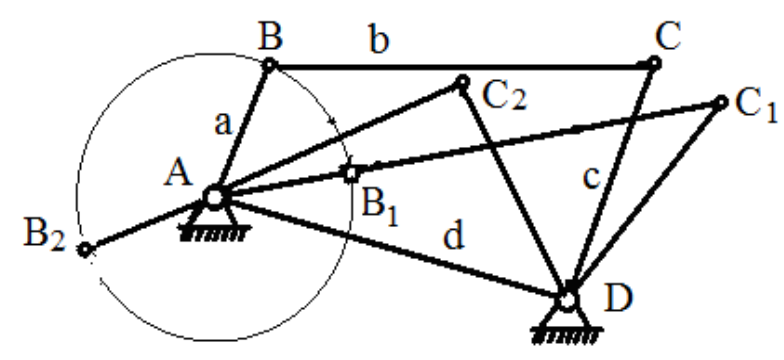

Figure 1: Design scheme of the articulated four link

To derive the condition for the existence of the crank, we consider the articulated four-link in its two extreme positions (Figure 1). It is known that in the extreme positions of the lever mechanism, the axis of the crank and connecting rod lie on one straight line. The diagram in Fig. 1 shows the four-link $\mathrm{ABCD}$ in its extreme positions: $\mathrm{AB}_{1} \mathrm{C}_{1} \mathrm{D}$ and $\mathrm{AB}_{2} \mathrm{C}_{2} \mathrm{D}$. Denote the dimensions of the links, including the rack $\mathrm{AD}$, respectively, by a, b, c, d. Then, by the property of the triangle, that the sum of its two sides is greater than the third, we write the equations:

$$
\begin{aligned}
& \text { of } \triangle \mathrm{AC}_{1} \mathrm{D} \rightarrow \mathrm{d}<\mathrm{b}+\mathrm{a}+\mathrm{c} \\
& \text { of } \Delta \mathrm{AC}_{2} \mathrm{D} \rightarrow \mathrm{d}<\mathrm{b}-\mathrm{a}+\mathrm{c}
\end{aligned}
$$

These inequalities allow us to obtain the following:

$$
\begin{aligned}
& a+d<c+b(4) \\
& d-a<c+b(5)
\end{aligned}
$$

The fulfillment of inequality (4) ensures the fulfillment of inequality (5) regardless of the aspect ratio $c$ and b. And if inequality (5) is satisfied at the maximum value of the left side, that is, when $d$ is the size of the longest link, and a is the size of the shortest, then link a will be a crank and will be able to complete a full revolution around the rack. This conclusion is the essence of the Grashof rule: the crank can be the shortest link of the articulated four link, if the sum of the lengths of the shortest and longest links is less than the sum of the lengths of the remaining links [4].

\section{RESULTANDDISCUSSION}

The task of the kinematic analysis of cam mechanisms is to determine the displacements $S$, velocities $\vartheta$, accelerations $\alpha$ of the points of the driven link according to a given outline of the cam profile (phase angles), the law of cam movement and the kinematic scheme of the mechanism (Figure 2).

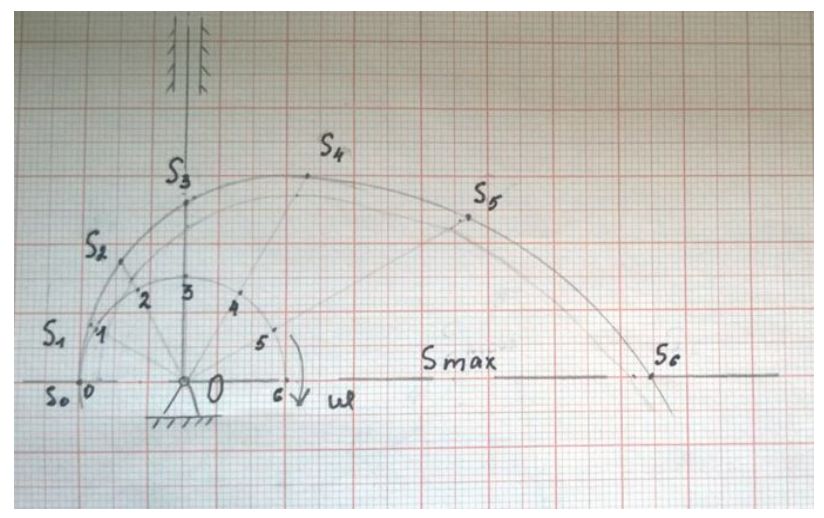

Figure 2: Cam mechanism with roller follower. 
To build a diagram of the displacement of the point $S$ of the slider, we postpone along the abscissa the segment $\ell=$ $120 \mathrm{~mm}$, depicting the period $\mathrm{T}$ of one turn of the crank, and divide it into 12 equal parts (Figure. 3). From points 1 , $2 \ldots \ldots 11$ of the diagram $\mathrm{S}(\mathrm{t})$, we put the ordinates $1-1,2-$ $2 \ldots, 11-11$, respectively, equal to the distances S0 - S1 S0 - S2. ..... S0 - S12 traversed by point S from the origin.

We calculate the scales of displacement:

$$
\begin{gathered}
\mu_{S}=\frac{S_{\text {max }}}{H_{\text {max }}}=\frac{70 \mathrm{MM}}{55 \mathrm{MM}}=1,27 \\
\mu_{t}=\frac{T}{L}=\frac{60}{120 * 120}=0,0042(\mathrm{~s} / \mathrm{mm}) \\
\mu_{\varphi}=\frac{2 \pi}{L}=\frac{2 * 3,14}{120}=0,0523(\mathrm{rad} / \mathrm{mm})
\end{gathered}
$$

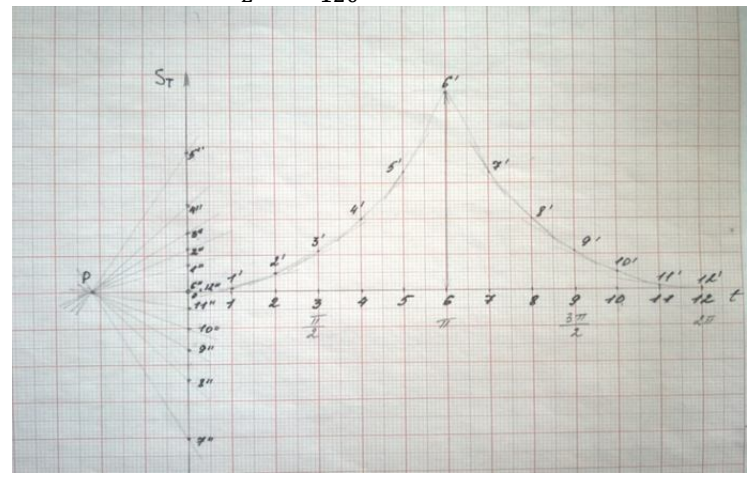

Figure 3: Cam point $S$ movement diagram

The speed diagram is constructed by graphically differentiating the movement graph using the chord method.

To construct it, we replace the curved sections of the graph $\mathrm{S}(\mathrm{t})$ with straight lines $0-1$ ', 1'-2'... 11'-12'. Under the displacement graph, draw the rectangular axes v and t. On the $\mathrm{t}$ axis, select the pole distance $\mathrm{H}=22 \mathrm{~mm}$. From the pole $\mathrm{p}$ we draw the inclined straight lines $\mathrm{p}-1$ ', $\mathrm{p}-2$ '... $\mathrm{p}$ 11 'parallel to the chords $0-1$ ', 1 ' -2 ' ... 11'-0. From the middle of the intervals $0-1,1-2 \ldots 11-0$ of the diagram $v(t)$ we draw perpendiculars to the $t$ axis. From points 1 ', 2' ... 11 ', we draw straight lines parallel to the $t$ axis. Connect the intersection points with a smooth curve (Figure 4).

The scale of the speed diagram is calculated by the

$$
\text { formula: } \mu_{v}=\frac{\mu_{s}}{\mu_{t} \cdot H}=\frac{1,27}{0,0042 \cdot 55}=5,498\left(s^{-1}\right)
$$

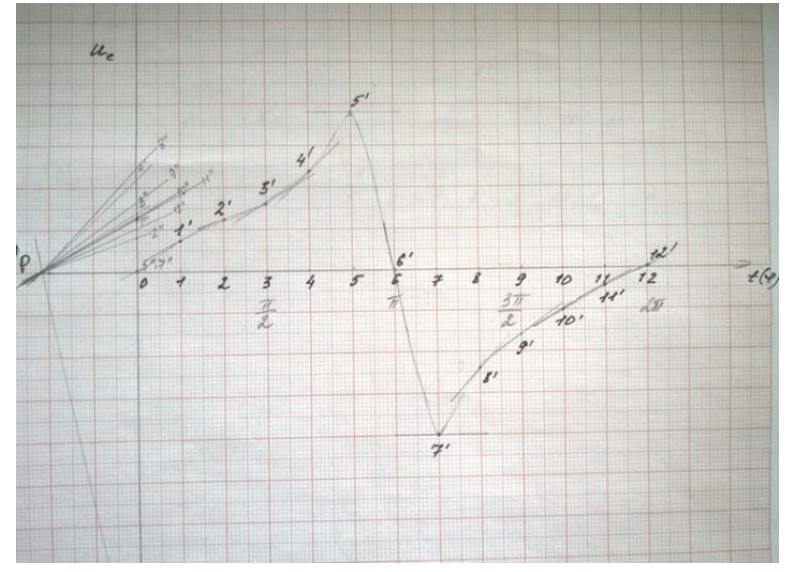

Figure 4: S point velocity diagram
The construction of the corresponding cam profile element for the central mechanism with a roller follower is carried out as follows:

1) From the center $O$ of rotation of the cam, draw a circle of radius $r_{0}+\rho$.

For this mechanism:

$$
\begin{gathered}
r_{0}=25 \mathrm{~mm} \\
\rho=3 \mathrm{~mm} \\
\text { then } r_{0}+\rho=28 \mathrm{~mm}
\end{gathered}
$$

From the radial line OSO corresponding to the initial position of the pusher, we postpone in the opposite direction, the angle of rotation of the $\operatorname{cam} \varphi=90^{\circ}$, which is divided into 11 parts [7].

Given the scale factor, which is determined by the formula tables 1

Weget:

$$
\mu_{v}=\frac{90}{11}=8,18(\mathrm{rad} / \mathrm{mm})
$$

Tables 1:scale factor

\begin{tabular}{|c|c|}
\hline$\varphi_{1}$ & $8,18^{\circ}$ \\
\hline$\varphi_{2}$ & $16,36^{\circ}$ \\
\hline$\varphi_{3}$ & $24,54^{\circ}$ \\
\hline$\varphi_{4}$ & $32,72^{\circ}$ \\
\hline$\varphi_{5}$ & $40,9^{\circ}$ \\
\hline$\varphi_{6}$ & $49,08^{\circ}$ \\
\hline$\varphi_{7}$ & $57,26^{\circ}$ \\
\hline$\varphi_{8}$ & $65,44^{\circ}$ \\
\hline$\varphi_{9}$ & $73,62^{\circ}$ \\
\hline$\varphi_{10}$ & $81,8^{\circ}$ \\
\hline$\varphi_{11}$ & $90^{\circ}$ \\
\hline
\end{tabular}

1) We determine the scale by the formula:

$$
\mu_{s}=\frac{h_{\max }}{y_{\max }}=\frac{70}{50}=1,4
$$

2) From the circumference of a radius of $28 \mathrm{~mm}$ drawn through the initial position of the center of the roller, we postpone the values of the displacement of the pusher along the radial lines (taking into account the scale) tables 2. [3].

Tables 2: values of the displacement of the pusher along radial lines (taking into account the scale):

\begin{tabular}{|c|c|}
\hline $\mathbf{S}_{\mathbf{1}}$ & $1,4 \mathrm{~mm}$ \\
\hline $\mathbf{S}_{\mathbf{2}}$ & $5,6 \mathrm{~mm}$ \\
\hline $\mathbf{S}_{\mathbf{3}}$ & $13,3 \mathrm{~mm}$ \\
\hline $\mathbf{S}_{\mathbf{4}}$ & $23,8 \mathrm{~mm}$ \\
\hline $\mathbf{S}_{\mathbf{5}}$ & $36,4 \mathrm{~mm}$ \\
\hline $\mathbf{S}_{\mathbf{6}}$ & $49 \mathrm{~mm}$ \\
\hline $\mathbf{S}_{\mathbf{7}}$ & $58,8 \mathrm{~mm}$ \\
\hline $\mathbf{S}_{\mathbf{8}}$ & $65,1 \mathrm{~mm}$ \\
\hline $\mathbf{S}_{\mathbf{9}}$ & $67,9 \mathrm{~mm}$ \\
\hline $\mathbf{S}_{\mathbf{1 0}}$ & $69,3 \mathrm{~mm}$ \\
\hline $\mathbf{S}_{\mathbf{1 1}}$ & $70 \mathrm{~mm}$ \\
\hline
\end{tabular}

3) The resulting points $\mathrm{S} 0, \mathrm{~S} 1, \mathrm{~S} 2, \mathrm{~S} 3, \ldots \mathrm{S} 11$ are connected by a smooth curve (theoretical cam profile) (Figure 5).

4) To build a practical profile from any points located on the theoretical profile, draw arcs of circles of radius equal 
to the radius of the roller $\mathrm{r}=3 \mathrm{~mm}$. The envelope internal to these arcs is the true (practical) cam profile.

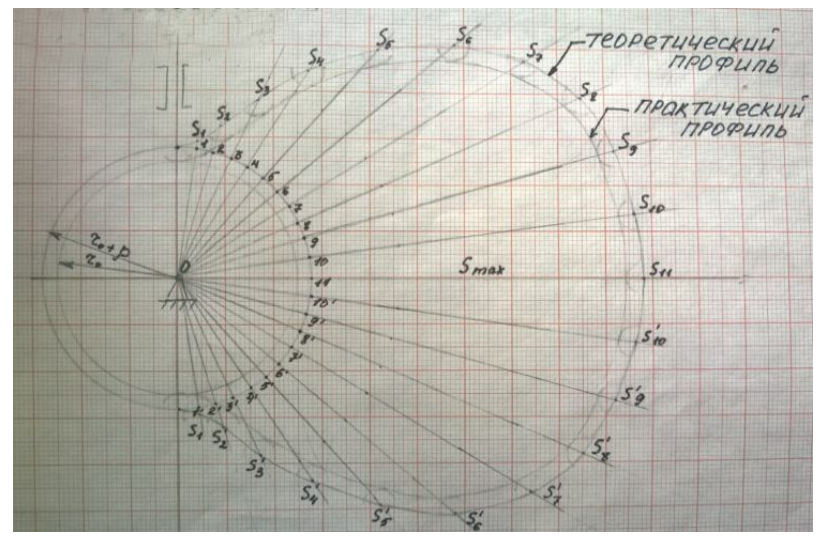

Figure 5: Cam Profile

\section{CONCLUSION}

Thus, as a study, a kinematic analysis of the flat mechanism was carried out, namely: speed and acceleration plans were constructed for each of the five positions of this mechanism. The trajectories of points for eight positions of this mechanism are constructed. An analysis of the cam mechanism by the method of diagrams, the profile of the cam is built.

\section{REFERENCES}

1. Bertyaev V.D., Bulatov L.A., Komolov D.V., Markelov S.S. Kinematic calculation of a flat multi-link mechanism. - Tula, 2003;

2. Filippov A.A., Pachurin G.V., Goncharova D.A., Mukhina M.V., Katkova O.V., Chaikina Z.V., Smirnova J.V., Tsapina T.N. Resource efficient preparation of high quality rolled stock for motor vehicles fastening // In collection: IOP Conference Series: Materials Science and Engineering. 2019.S. 012078.

https://doi.org/10.1088/1757-899X/560/1/012078

3. Pachurin G.V., Shevchenko S.M., Mukhina M.V., Kutepova L.I., Smirnova J.V.The factor of structure and mechanical properties in the production of critical fixing hardware 38XA // Tribology in Industry. 2016.V. 38. No. 3. P. 385-391.

4. Kutepova L.I., Parshakova A.M. Modern computer technology in the design of linear structures and engineering surveys //

5. Cherney O.T., Suldina V.V. Heat treatment as part of the manufacturing process of manufacturing engineering parts // In the collection: Integration of information technology in the system of professional and additional education. collection of articles on the materials of the IV regional scientific-practical conference. Nizhny Novgorod State Pedagogical University named after KozmaMinin. 2018.S. 61-63.

6. Y. Pratapa Reddy, Dr. K. L Narayana, Dr. M. Kedar Mallik. Electro-chemical behavior of different metals in Sodium Chloride solution // International Journal of Emerging Trends in Engineering Research Volume 7, No. November 11, 2019. https://doi.org/10.30534/ijeter/2019/507112019

7. Smirnova Zh.V, Cherney O.T. Laser alloying technology when changing the processing speed // International Journal of Emerging Trends in Engineering Research. Volume 8. No. April 4, 2020

https://doi.org/10.30534/ijeter/2020/40842020

8.Yatsun, S.F. Kinematics, dynamics and strength of machines, instruments and equipment: a training manual [Text]: S.F. Yatsun, V.Ya. Mishchenko, E.N. Politov - M: Alpha-M: INFRA-M, 2012.-208s. 